\title{
A Miniaturized MEMS Motion Processing System for Nuclear Medicine Imaging Applications
}

\author{
Mojtaba Jafari Tadi ${ }^{1,2}$, Eero Lehtonen ${ }^{2}$, Jarmo Teuho ${ }^{1}$, Antti Saraste ${ }^{1,3}$, Mikko Pänkäälä ${ }^{2}$, Mika \\ Teräs $^{1,3}$, Tero Koivisto ${ }^{2}$ \\ ${ }^{1}$ Turku PET Center, Turku, Finland \\ ${ }^{2}$ Technology Research Center, Turku, Finland \\ ${ }^{3}$ University of Turku Hospital, Turku, Finland
}

\begin{abstract}
Cardiac, respiratory, and patient body motion artifacts degrade the image quality and quantitative accuracy of the nuclear medicine imaging which may lead to incorrect diagnosis, unnecessary treatment and insufficient therapy. We present a new miniaturized system including joint micro electromechanical (MEMS) accelerometer and gyroscope sensors for simultaneous extraction of cardiac and respiratory signals. We employ two tri-axial joint MEMS sensors for selecting an optimal trigger point in a cardiac and respiratory cycle. The 6-axis motion sensing helps to detect candidate features for cardiac and respiratory gating in Positron emission tomography (PET) imaging. The aim of this study was to validate MEMS-derived signals against traditional Real-time Position Management $(R P M)$ and electrocardiography (ECG) measurement systems in 4 healthy volunteers. High agreement and correlation were found between cardiac and respiratory cycle intervals. These promising first results warrant for further investigations.
\end{abstract}

\section{Introduction}

In cardiac and oncologic Positron emission tomography (PET)/computed tomography (CT) imaging, cardiac, respiratory, and patient body motions may impair the image quality and the quantitative accuracy of heart imaging $[1-3]$. To reduce motion-related inaccuracies, cardiac and respiratory gating methods are the most common approaches applied in clinical PET imaging [4]. Simultaneous respiratory and cardiac gating, namely dual gating, can reduce motion-related inaccuracies and correspondingly imaging resolution in cardiac PET and oncological applications [5]. Cardiac gating is accomplished by an electrocardiography (ECG) measurement system, while respiratory gating can be performed by external devices such as spirometry, elastic belts (consisting of pressure or load cell sensors) monitors or using optical techniques including a camera and laser sensor that track chest wall or abdomen displacement $[3,6,7]$. However, respiratory gating devices have been considered for only research purposes due to the need for complex logistics and long data processing which may increase patient discomfort and be laborious for the clinicians [2,3]. Additionally, ECG is able to show only electrical activity of the heart and still fails to trace the instantaneous mechanical state of the heart due to the stirring movements of the myocardium [8]. Thus, these challenges complicate PET imaging protocols and create a serious demand for simultaneous recording of cardiac and respiratory signals in dual gating. Recently, new techniques based on bioimpedance [2,9] and accelerometers [10] have been suggested for concurrent acquisition of both respiratory and cardiac signals in oncologic and cardiac PET imaging. However, there is still space to optimize these methods, especially in terms of technology, accuracy, and patient comfort.

We present a new framework based upon tri-axial microelectromechanical (MEMS) accelerometer and gyroscope sensors to extract cardiac and respiratory signals. Our main objective in this study is to validate MEMS motion processing configuration against real-time position management and electrocardiography (ECG) measurement systems. Our hypothesis is that mechanical sensors could be used improve the quality of detection and estimation of the quiescent periods in cardiac and respiratory cycles. Since pure chest wall displacement due to respiration and heart motion can be monitored using mechanical sensors, MEMS-based measurements offer a potential method for nuclear medicine imaging applications.

\section{Materials and Methods}

Currently, there is no such miniaturized device designed for dual gating which is able to demonstrate electromechanical and respiratory motions in PET/CT imaging, 


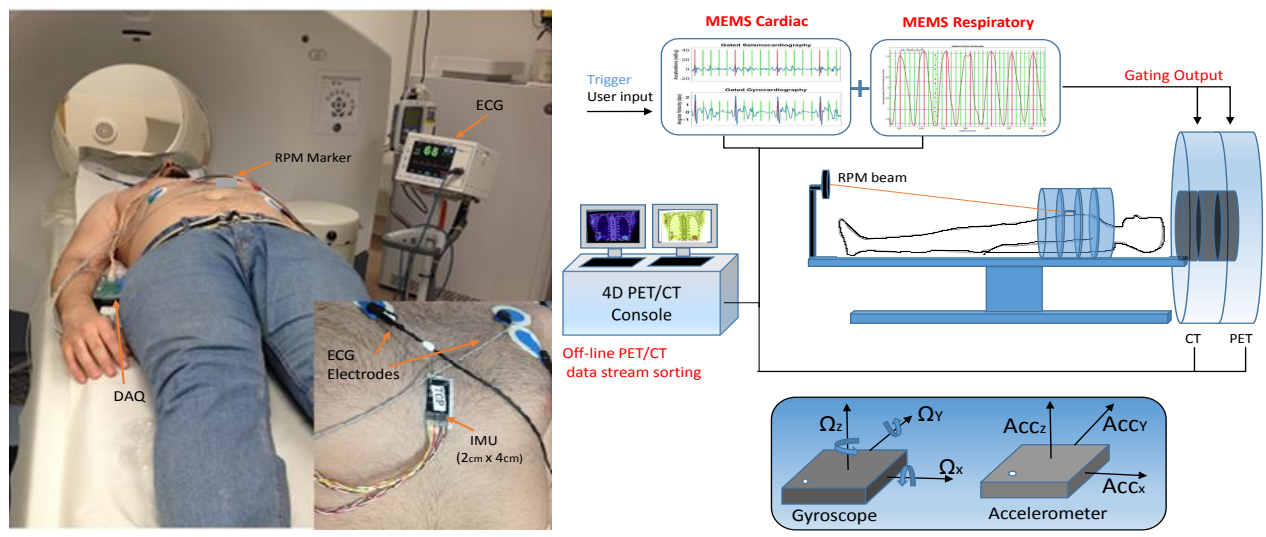

Figure 1. Left: The diagram demonstrates the measurement set up including data acquisition (DAQ) unit, the IMU attached to the subject chest, two lead ECG, and the RPM recording. Right: A simplified block diagram of 6-axis MEMS dual gating system for PET/CT imaging. Arrows show orientation of sensitivity and the polarity of 6-axis accelerometer and gyroscope sensors. This diagram is partially modified from GE healthcare white paper for demonstration purposes.

simultaneously. Available real-time position management (RPM) systems require the use of camera and markers. However, inaccurate placement of a RPM marker block on the targeted location is a source of error itself. This study employs a miniaturized joint accelerometergyroscope system, or namely inertial motion unit (IMU), in order to measure the heart motions and longitudinal displacement of the chest. Toward this end, a $(3 \mathrm{~mm} \times$ $3 \mathrm{~mm} \times 1 \mathrm{~mm}$ ) triple-axis, low-power, capacitive digital accelerometer (Freescale Semiconductor, MMA8451Q, Austin, TX, USA $)$ and an $(3 \mathrm{~mm} \times 3 \mathrm{~mm} \times 0.9 \mathrm{~mm})$ ultra-accurate, low power, low noise, 3 -axis angular rate sensor (Maxim Integrated, MAX21000, San Jose, CA, U.S.) were employed for the upper chest motion processing purposes. Accelerometer-based seismocardiography (SCG) measures the linear motions of the chest wall, while the gyrocardiography (GCG) measures the angular motions [11]. Concurrent 10 minute recordings of MEMSbased SCG and GCG, electrocardiography (Texas Instruments ADS1293), and Real-time Position Management (from Varian Medical Systems, Palo Alto, CA, USA ) were performed, processed and analysed using four healthy volunteers as test subjects.

Signal processing algorithms for extracting gating information from cardiac and respiration signals were developed. The MEMS based measurements, RPM and ECG recordings were accurately aligned using cross correlation techniques. An adaptive algorithm based upon Hilbert transform was used that autonomously detects the heart beat's position and calculates beat-to-beat intervals [12]. The position of heart beats can be determined from the particular wave forms in the SCG and GCG repeating with every heartbeat. In addition to cardiac signals, the respiration waveforms due to the longitudinal displacements of the chest were extracted using a brick-wall bandpass filter [0.1-1 Hz], smoothed using a moving average filter, and finally normalized based upon successive mean quantization transform [10]. The gyroscope signal were also integrated so that chest longitudinal displacement was achieved. In practice, accelerometer derived respiration (ADR) and gyroscope derived respiration (GDR) can be considered for respiratory gating and three axis SCG and GCG (together 6-axis) cardiac signals can be used for cardiac gating.

Gating simply means dividing the acquired image into individual time-stamped bins that correlate to phases of respiratory and/or cardiac motion by overlaying corresponding time-stamped data [4]. The input signal in the clinical PET/CT image reconstructions based upon gating techniques includes trigger markers indicating the onset of systole and diastole phases on a cardiac signal or inspiration and expiration on a respiration signal. Fig. 1 shows a simplified diagram of the proposed method for dual gating based on MEMS sensors.

\section{Results}

The recorded ECG and MEMS signals were processed off-line. In the ECG signal, the QRS complexes were detected using the Pan-Tompkins method [13]. The heart rate detection algorithm described in [12] was applied to the SCG and GCG signals separately. The performance of the cardiac and respiratory cycle estimations was assessed and computed separately for each subject by means of different statistical parameters such as Pearson correlation, root mean square error (RMSE) and $95^{\text {th }}$ percentile rate. The algorithms efficiency with respect to the cycle intervals and detected heart beats was evaluated against a synchronized lead II ECG as reference. Similarly, the respiratory cy- 

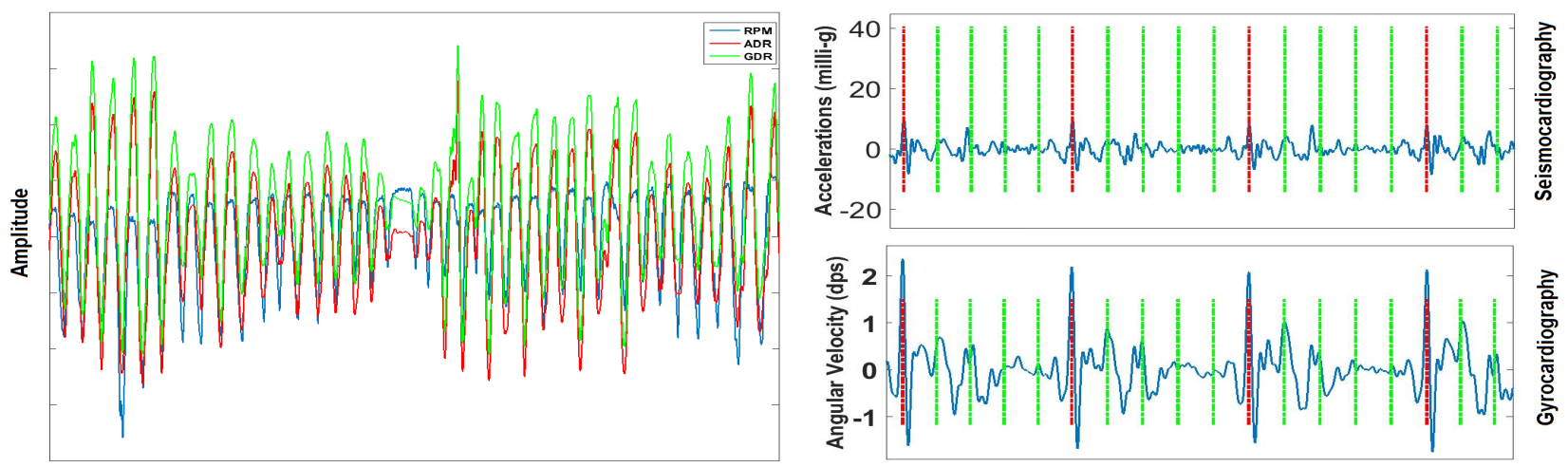

Figure 2. The diagram demonstrates the accelerometer- and gyroscope-derived respirations against RPM measurement (left inset). The right insets show the SCG and GCG signals for prospective gating applications.

cles estimated by ADR and GDR measures were evaluated against RPM signals.

Accordingly, the cardiac cycles detected with the MEMS-based SCG and GCG measurements are highly correlated with the reference ECG cycles in terms of interval durations ( $\mathrm{R} 1_{G C G v s . E C G}=0.998, \mathrm{R} 2_{S C G v s . E C G}$ $=0.999)$. Additionally, the corresponding average error rates ( RMSE, 95 ${ }^{\text {th }}$ percentile) for SCG and GCG interbeat intervals against ECG were $(0.227 \mathrm{~ms}, 0.36 \mathrm{~ms}$ ) and $(0.196 \mathrm{~ms}, 0.429 \mathrm{~ms})$, respectively. This implies that mechanical signals are able to accurately indicate the cardiac cycle intervals suitable for cardiac gating, as compared to the ECG. Moreover, the gyroscope and accelerometer derived respiratory signals (GDR and ADR) are found to have high linear correlation with the reference RPM (R1 $\left.1_{G D R v s . R P M}=0.991, \mathrm{R} 2_{A D R v s . R P M}=0.987\right)$. The corresponding average error rates ( RMSE, $95^{\text {th }}$ percentile) for the GDR and ADR cycle intervals against RPM were $(17.64,35.14 \mathrm{~ms})$ and $(17.32,35.51 \mathrm{~ms})$, respectively. Table 1 and Table 2 show the results of statistical analysis in this study.

\section{Discussion}

The aim of this study was to test a miniaturized MEMS motion processing system for nuclear medicine imaging applications by detecting and segregating of the cardiac and respiratory motion signals instantaneously. A hardware system which was able to obtain synchronous gyrocardiography, seismocardiography, and electrocardiography was used to implement this study. In our study we propose a novel non-invasive technique to assess cardiac and respiratory motions by miniaturized tri-axial MEMS accelerometer and gyroscope sensors attached to surface of the human chest. In practice, gyrocardiogram is less noisy than seismocardiogram due to the following reasons. Accelerometer is very susceptible to earth gravity which makes it more prone to vibration/noise. Whereas, gyroscope is not affected by the gravity of earth and is insusceptible to axial and radial translational motions [14]. Additionally, gyroscope is an active sensor that consumes more power than accelerometer which is a passive sensor. Thus, higher power consumption in the case of gyroscope may result in high signal to noise ratio (SNR) where there is low SNR in accelerometer.

Table 1. Correlation test and error analysis between MEMS cardiac signals and reference ECG (indexes 1 and 2 refer to GCG and SCG, respectively.)

\begin{tabular}{|c|l|l|l|l|l|l|}
\hline ID & R1 & R2 & $\begin{array}{l}\text { RMSE1 } \\
(\mathrm{ms})\end{array}$ & $\begin{array}{l}\text { E95_1 } \\
(\mathrm{ms})\end{array}$ & $\begin{array}{l}\text { RMSE2 } \\
(\mathrm{ms})\end{array}$ & $\begin{array}{l}\text { E95_2 } \\
(\mathrm{ms})\end{array}$ \\
\hline 1 & 0.999 & 0.998 & 0.013 & 0.004 & 0.077 & 0.062 \\
\hline 2 & 0.999 & 1 & 0.015 & 0.004 & 0.003 & 0.004 \\
\hline 3 & 0.996 & 1 & 0.653 & 1.072 & 0.509 & 1.222 \\
\hline 4 & 0.999 & 0.998 & 0.003 & 0.006 & 0.059 & 0.107 \\
\hline $\bar{\alpha}$ & 0.998 & 0.999 & 0.227 & 0.360 & 0.196 & 0.429 \\
\hline
\end{tabular}

Table 2. Correlation test and error analysis between MEMS derived respiration signals and RPM (indexes 1 and 2 refer to GCG and SCG, respectively.)

\begin{tabular}{|c|l|l|l|l|l|l|}
\hline ID & R1 & R2 & $\begin{array}{l}\text { RMSE1 } \\
(\mathrm{ms})\end{array}$ & $\begin{array}{l}\text { E95_1 } \\
(\mathrm{ms})\end{array}$ & $\begin{array}{l}\text { RMSE2 } \\
(\mathrm{ms})\end{array}$ & $\begin{array}{l}\text { E95_2 } \\
(\mathrm{ms})\end{array}$ \\
\hline 1 & 0.998 & 0.998 & 13.30 & 24.88 & 13.85 & 31.28 \\
\hline 2 & 0.997 & 0.998 & 11.34 & 22.32 & 9.35 & 19.2 \\
\hline 3 & 0.997 & 0.996 & 16.29 & 39.12 & 20.90 & 34.32 \\
\hline 4 & 0.970 & 0.956 & 29.65 & 54.25 & 25.12 & 45.25 \\
\hline $\bar{\alpha}$ & 0.991 & 0.987 & 17.64 & 35.14 & 17.30 & 32.51 \\
\hline
\end{tabular}

This research investigated whether a joint 6-axis accelerometer-gyroscope unit has the potential to prepare the suffice dual gating information for the cardiac PET imaging. This study concentrated on validating the MEMS based measurement against different reference systems (RPM and ECG). As results were shown in this study, a high linear correlation was observed between both cardiac and respiratory MEMS measurements and reference systems. Fig. 2 shows how well the MEMS derived respi- 
ratory signals mimic the reference RPM signal. Furthermore, GCG represents high quality cardiac motion signals which warrants the capability of MEMS gating for PET/CT imaging applications. However, there are some limitations in this study such as the following. Only healthy volunteers were examined while real cardiac or oncological patients are excluded. In future, the proposed method can be employed in clinical cardiac or oncology examinations for example PET/CT imaging. Thus, further studies are required in order to tackle technical challenges with the MEMS gating study.

\section{Conclusion}

The proposed MEMS motion sensor-based gating, and the developed algorithms, may potentially improve the accuracy of nuclear medicine imaging specifically in cardiovascular and oncologic studies. It would be helpful for clinical studies since both gating signals can be acquired with a single, miniaturized, and non-invasive MEMS device. Overall, the proposed system might offer motionless images with the lower radiation dose. Having a high resolution cardiac image definitely helps a heart specialist or oncologist in making a correct diagnosis as well as planning a sufficient therapy. Therefore, these promising first results warrant for further investigations, and the application of the developed methods on real PET/CT image reconstruction.

\section{Acknowledgements}

The authors would like to thank the staff at Turku PET center for excellent assistance during the experiments in this work and Technology Research Center for their efforts in designing the prototype measurement system. This work was supported in part by Academy of Finland under Grant 277383 and by the University of Turku Graduate School.

\section{References}

[1] Teräs M, Kokki T, Durand-Schaefer N, Noponen T, Pietilä M, Kiss J, Hoppela E, Sipilä HT, Knuuti J. Dual-gated cardiac PET-clinical feasibility study. European Journal of Nuclear Medicine and Molecular Imaging 2009;37(3):505516. ISSN 1619-7089.

[2] Koivumäki T, Vauhkonen M, Kuikka JT, Hakulinen MA. Bioimpedance-based measurement method for simultaneous acquisition of respiratory and cardiac gating signals. Physiological Measurement 2012;33(8):1323.

[3] Slomka PJ, Pan T, Germano G. Imaging moving heart structures with PET. Journal of Nuclear Cardiology 2016; 23(3):486-490. ISSN 1532-6551.

[4] Kokki T, Sipilä HT, Teräs M, Noponen T, Durand-Schaefer N, Klén R, Knuuti J. Dual gated PET/CT imaging of small targets of the heart: Method description and testing with a dynamic heart phantom. Journal of Nuclear Cardiology 2009;17(1):71-84. ISSN 1532-6551.

[5] Klein GJ, Reutter BW, Huesman RH. Non-rigid summing of gated PET via optical flow. In Nuclear Science Symposium, 1996. Conference Record., 1996 IEEE, volume 2. ISSN 1082-3654, Nov 1996; 1339-1342 vol.2.

[6] Nehmeh SA, Erdi YE, Pan T, Yorke E, Mageras GS, Rosenzweig KE, Schoder H, Mostafavi H, Squire O, Pevsner A, Larson SM, Humm JL. Quantitation of respiratory motion during 4dPET/CT acquisition. Medical Physics 2004;31(6).

[7] Kokki T, Klén R, Noponen T, Pärkkä J, Saunavaara V, Hoppela E, Teräs M, Knuuti J. Linear relation between spirometric volume and the motion of cardiac structures: MRI and clinical PET study. Journal of Nuclear Cardiology 2016;23(3):475-485. ISSN 1532-6551.

[8] Wick C, Su JJ, McClellan J, Brand O, Bhatti P, Buice A, Stillman A, Tang X, Tridandapani S. A system for seismocardiography-based identification of quiescent heart phases: Implications for cardiac imaging. Information Technology in Biomedicine IEEE Transactions on Sept 2012;16(5):869-877.

[9] Koivumäki T, Nekolla SG, Frst S, Loher S, Vauhkonen M, Schwaiger M, Hakulinen MA. An integrated bioimpedanceecg gating technique for respiratory and cardiac motion compensation in cardiac PET. Physics in Medicine and Biology 2014;59(21):6373.

[10] Jafari Tadi M, Koivisto T, Pänkäälä M, Paasio A. Accelerometer-based method for extracting respiratory and cardiac gating information for dual gating during nuclear medicine imaging. International Journal of Biomedical Imaging 2014;2014(690124):1-11.

[11] Jafari Tadi M, Lehtonen E, Pänkäälä M, Saraste A, Vasankari T, Teräs M, Koivisto T. Gyrocardiography: a new non-invasive approach in the study of mechanical motions of the heart. concept, method and initial observations. In Engineering in Medicine and Biology Society, EMBC, 2016 Annual International Conference of the IEEE. Aug 2016 ; $1-4$.

[12] Jafari Tadi M, Lehtonen E, Hurnanen T, Koskinen J, Eriksson J, Pänkäälä M, Teräs M, Koivisto T. A real-time approach for heart rate monitoring using hilbert transform in seismocardiograms. Physiological Measurement 2016; 37(10):1-25.

[13] Pan J, Tompkins WJ. A Real-Time QRS Detection Algorithm. Biomedical Engineering IEEE Transactions on March 1985;BME-32(3):230-236.

[14] Marcelli E, Cercenelli L, Parlapiano M, Fumero R, Bagnoli P, Costantino ML, Plicchi G. Effect of right ventricular pacing on cardiac apex rotation assessed by a gyroscopic sensor. American Society for Artificial Internal Organs 2007; 53(3):304-309.

Address for correspondence:

Mojtaba Jafari Tadi, Turku PET Center

University of Turku, Finland

mojtaba.jafaritadi@utu.fi 